\title{
The Effect of Vaccination Decision in Disease Modelling through Simulation
}

\author{
Teoh Shian Li, Jane Labadin, Phang Piau, Shapiee Abd Rahman, Ling Yeong Tyng \\ Department of Computational Science and Mathematics \\ Faculty of Computer Science and Information Technology, Universiti Malaysia Sarawak \\ 94300 Kota Samarahan, Sarawak, Malaysia \\ shianli90@gmail.com, ljane@pps.unimas.my, pphang@fit.unimas.my, sar@fit.unimas.my, ytling@fit.unimas.my
}

\begin{abstract}
Infectious disease is one of the health issues that threatens the world population. The spread of infectious diseases can be controlled via vaccination program and self-isolation as suggested by the public health organization. The individuals' incentives to take the control measures are depend on their fearfulness on the disease. This fearfulness or feeling of fear is associated with human behavior. Hence, the objective of this study is to incorporate human behavior in disease modeling. We also aimed to study the level of fearfulness in both subpopulations of receiving complete information and receiving incomplete information and its impact to the vaccination program. In this paper, a model is developed by using individualbased modelling approach to capture the human behavioral changes during disease outbreak. The analysis of the results presented the relationship between the fearfulness and the vaccination decision of the individual. Hence, the level of fearfulness needs to be examined in order to organize an effective vaccination program.
\end{abstract}

Keywords-infectious disease modelling; individual-based modelling; human behavioral changes; vaccination decision; imitation dynamics

\section{INTRODUCTION}

Infectious diseases have been recognized as one of the crucial issues that threaten the health of the world population. It is defined as the illness which is induced by the pathogenic organisms such as viruses, fungi or protozoa [1]. There are a lot of factors that affect the spread of infectious diseases, namely geographical, environmental, and demographical. Many of the epidemiological characteristics are taken into consideration in disease modelling, for instance, the latent period, disease transmission rate, and recovery rate for the diseases [2]. Back in 1927, Kermack-McKendrick model marked the beginning of the mathematical disease modelling which considered the disease transmission rate and the recovery rate for the disease [3]. This model is governed by three ordinary differential equations (ODEs). There are three compartments in this model, namely Susceptible, Infected, and Recovered, which represent the subpopulations accordingly. The basic SusceptibleInfected-Recovered (SIR) model considers only of viruses' transmission and recovery rates. It does not take into account of other factors.

Besides, human behavior also affects the spread of disease. Thus, human behavioral changes have been taken into account in recent disease modelling [4]. The interaction among the individuals in a population and how they behave during the disease outbreak play an important role in epidemiology as they influence the dynamic of the disease spread. During the disease outbreak, mass media information about this disease will be spread widely. It will then induce fearfulness and awareness in the population. Fearfulness can be expressed as a social anxiety contagious process [5]. It can be spread in the population due to the interactions of the individuals. If an individual is in fear of the disease, he/she may spread the fearfulness by influencing his/her neighbors. The neighbors here are referred to the people surrounding him/her, for instance, family and close friends. Fig. 1 shows a simple illustration on how the fearfulness is spread from a feared individual. With the advanced telecommunications nowadays, the fearfulness not only can be spread through physical contacts, but also via the social networks, for instance, Twitter. Twitter can be used as a digital surveillance of the spread of fearfulness during a disease outbreak [6]. The fearfulness is spread to the friends in the social circle via the tweets posted. As mentioned before, the information of the disease induces fearfulness to the individuals. Some individuals are not exposed to the information coverage and hence they will receive less or incomplete information about the disease during the outbreak. Therefore, they will have lower awareness or fearfulness on the disease.

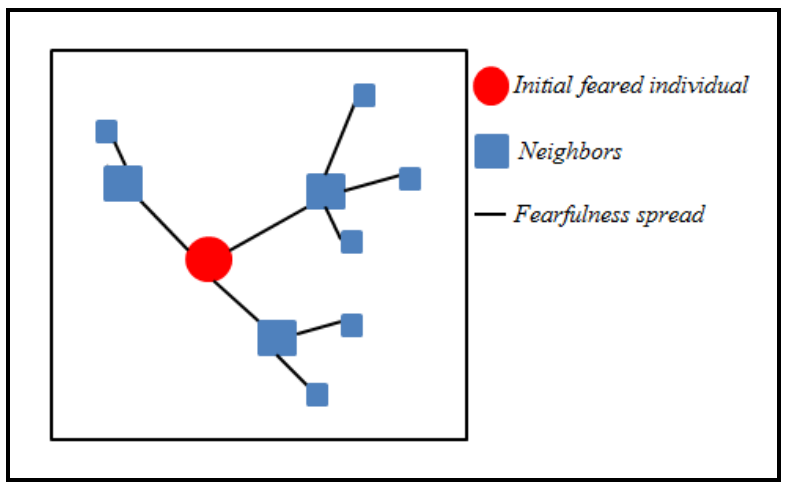

Fig. 1 Illustration of the spread of fearfulness.

When the individuals are feared of the disease, they have higher incentive to take the control measures. In order to prevent the infectious diseases to be spread, the authorities have recommended some precautions, for example, selfisolation during disease outbreak, vaccination program, and 